\title{
Transcriptional control of the B3GALT5 gene by a retroviral promoter and methylation of distant regulatory elements
}

\author{
Aida Zulueta,* Anna Caretti,* Paola Signorelli,* Fabio Dall'Olio, ${ }^{\dagger}$ \\ and Marco Trinchera ${ }^{\ddagger}, 1$ \\ *Department of Health Sciences, San Paolo Hospital, University of Milan, Milan, Italy; \\ ${ }^{\dagger}$ Department of Experimental, Diagnostic, and Specialty Medicine (DIMES), University of \\ Bologna, Bologna, Italy; and ${ }^{\ddagger}$ Department of Medicine Clinical and Experimental (DMCS), \\ University of Insubria, Varese, Italy
}

ABSTRACT We focused on transcription factors and epigenetic marks that regulate the B3GALT5 gene through its retroviral long terminal repeat (LTR) promoter. We compared the expression levels of the B3GALT5 LTR transcript, quantitated by competitive RT-PCR, with those of the candidate transcription factors HNF1 $\alpha / \beta$ and Cdx1/2, determined by Western blot analysis, in colon cancer biopsies, various cell lines, and cell models serving as controls. We found that $\mathrm{HNF} 1 \alpha / \beta$ were easily detected, irrespective of the amount of LTR transcript expressed by the source, whereas Cdx1/2 were undetectable, and no sample lacking HNF1 $\alpha / \beta$ expressed the LTR transcript. On transfection in proper host cells, both HNF1 $\alpha$ and HNF1 $\beta$ provided detectable LTR transcript, whereas shRNA-mediated silencing of HNF1 $\beta$ impaired transcription. Treating cells with $5^{\prime}$-aza- $2^{\prime}$-deoxycytidine (5AZA) strongly reduced expression, without affecting HNF1 $\alpha / \beta$, despite the lack of CpG islands in the LTR and proximal sequences. By electrophoresis mobility shift and luciferase reporter assays, the LTR promoter binding and activity did not correlate with the amounts of LTR transcript expressed in the cells and depended on the levels of the transcription factors. We conclude that $\mathrm{HNF} 1 \alpha / \beta$ are necessary but insufficient to activate and regulate B3GALT5 LTR transcription, which depends on unknown regulatory elements that are active when methylated and located outside of and far from the LTR promoter.-Zulueta, A., Caretti, A., Signorelli, P., Dall'Olio, F., Trinchera, M. Transcriptional control of the B3GALT5 gene by a retroviral promoter and methylation of distant regulatory elements. FASEB J. 28, 946-955 (2014). www.fasebj.org

\footnotetext{
Abbreviations: 5AZA, 5'-aza-2'-deoxycytidine; Cdx, caudalrelated homeobox; EMSA, electrophoresis mobility shift assay; HEK, human embryonic kidney; HNF1, hepatocyte nuclear factor 1; LTR, long terminal repeat; RT-PCR, reverse transcription-polymerase chain reaction; TSA, trichostatin A; UTR, untranslated region
}

Key Words: hepatocyte nuclear factor $1 \cdot$ colon cancer $\cdot$ type 1 chain carbohydrates $\cdot$ transposon

The B3GALT5 GENe CODEs for $\beta 1,3$ galactosyltransferase 5 , an enzyme responsible for the synthesis of type 1 chain carbohydrates in mammals. In humans, in particular, it participates in the biosynthesis of the histo-blood group antigens Lewis a (Gal $\beta 1-3[$ Fuc $\alpha 1-$ 4] GlcNAc), Lewis b (Fuc $\alpha 1-2$ Gal $\beta 1-3[$ Fuc $\alpha 1-$ 4]GlcNAc), and sialyl-Lewis a (NeuAc $\alpha 2-3 \mathrm{Gal} \beta 1-$ 3 [Fuc $\alpha 1-4]$ GlcNAc) (1). The latter is a specific selectin ligand $(2,3)$ constituting the epitope of the CA19.9 antigen, which circulates in the serum of some patients with cancer $(4,5)$. B3GALT5 cDNA was first cloned from the human colon adenocarcinoma cell line COLO-205. The mRNA that corresponds to the coding sequence is found in the epithelia of various gastrointestinal tissues and some related cell lines (1) and is strongly down-regulated in colon cancer (6). A single transcription start site has been recognized, and a $5^{\prime}$-flanking region has been found to act as a promoter. The hepatocyte nuclear factor 1 (HNF1) and caudalrelated homeobox $(\mathrm{Cdx})$ transcription factors have been thought to control B3GALT5 expression according to a model reported for the typical intestinal enzyme sucrase-isomaltase (7). Of note, it was found soon after that the entire exon 1 of this B3GALT5 transcript, as well as the putative $\mathrm{HNF} 1 / \mathrm{Cdx}$-binding motif in the $5^{\prime}$ flanking promoter region, belonged to a retroviral long terminal repeat (LTR) sequence (8), also present in Old World monkeys, but not in New World monkeys or mice. This finding dates the insertion of the transposon to $25-30 \times 10^{6} \mathrm{yr}$ ago, and suggests the existence of another ancestral promoter (9). We have characterized multiple B3GALT5 tran-

\footnotetext{
${ }^{1}$ Correspondence: University of Insubria, via J. H. Dunant 5, 21100, Varese, Italy. E-mail: marco.trinchera@uninsubria.it doi: 10.1096/fj.13-236273

This article includes supplemental data. Please visit http:// www.fasebj.org to obtain this information.
} 
scription initiation sites and cognate $5^{\prime}$-untranslated regions (UTRs), as well as distinct $5^{\prime}$ flanking regions that are active as promoters (10). Among them, the type A promoter, which is sensitive to the CCAATbinding factor (NF-Y) and is also active in mice, has been proposed as the native promoter. In humans, the type A promoter is located $\sim 42 \mathrm{kbp}$ upstream of the LTR sequence in the context of $\mathrm{CpG}$ islands and appears to be active in many epithelia, but it is weakly active and down-regulated in cancer through epigenetic mechanisms (11). The type B promoter, located $\sim 27 \mathrm{kbp}$ upstream of the LTR in humans, appears as a defective, weak promoter that has the same HNF1/Cdx binding sequence as the LTR promoter, but the opposite orientation, and is thus thought to be related to the successful insertion of the transposon (10). The type C promoter has been found to be a rather strong one, active in the small intestine only. It is located $\sim 17 \mathrm{kbp}$ upstream of the LTR sequence, and its binding sites for transcription factors remain unknown. All B3GALT5 transcripts driven by such promoters share a common 3 ' sequence, including exons 3 (untranslated) and 4 (spanning the entire coding sequence), and differ in their 5'-UTRs only. Reevaluation of the tissue distribution of the various B3GALT5 mRNAs (10) indicated that the LTR transcript is very highly expressed only in normal colon mucosa, whereas, in the other organs of the gastrointestinal tract, including the small intestine, it is present in lower amounts. Such an expression pattern is thus the opposite of that reported for sucraseisomaltase, which is much more active in the small intestine than in the colon (12). Moreover, it was also reported that COLO-205 cells, expressing extremely high levels of B3GALT5 mRNA, including the LTR transcript (refs. 1, 13 and present work), are devoid of any $\mathrm{Cdx}$ expression (14). These findings appeared to be incompatible with the proposed model of B3GALT5 transcription and prompted us to reevaluate the model in detail. In particular, we wanted to elucidate the role of candidate transcription factors and the presence of potential epigenetic marks and explain tissue-specific expression and regulation, mainly in the process of colon tumorigenesis. Moreover, we wanted a better understanding of the evolutionary stabilization of the transposon that occurred in humans and some primates. To achieve these aims, we compared the expression levels of the B3GALT5 LTR transcript, quantitated by competitive reverse transcriptionpolymerase chain reaction (RT-PCR), with those of the candidate transcription factors $\mathrm{HNF} 1 \alpha / \beta$ and Cdx1/2, determined by Western blot analysis, in colon cancer biopsies, various cell lines, and cell models serving as controls. The models included cells transfected with plasmids coding $\mathrm{HNF} 1 \alpha / \beta$ cDNAs or shRNAs or treated with the demethylating agent $5^{\prime}$-aza2 '-deoxycytidine (5AZA). We also evaluated the behavior of the LTR promoter in vitro, through electrophoresis mobility shift (EMSA) and luciferase reporter assays.

\section{MATERIALS AND METHODS}

\section{Cell lines, tissues, and cell treatments}

The human breast cancer cell lines MCF-7 and MDA-MB-231, the human gastric cell lines $\mathrm{MKN}-45$ and KATOIII, the human bile duct carcinoma cell line HuCC-T1, and the human colon cancer cell lines HT-29, HCT-15, COLO-205, and SW-1116 were cultured as reported previously $(10,11,13$, 15). The human breast cancer cell line MDA-MB-361 (a gift of Dr. Cristina Razzari, University of Milan, Milan, Italy), the human embryonic kidney (HEK) cell line HEK-293T/17 [American Type Culture Collection (ATCC) CRL-11268, a gift of Dr. Anna Menini, Scuola Internazionale Superiore di Studi Avanzati (SISSA), Trieste, Italy], and the human hepatoma cell lines Huh-7 and Hep-3B (a gift of the GIM Microscopy Group; University of Milan), were cultured in DMEM containing $10 \%$ fetal bovine serum, $100 \mathrm{U} / \mathrm{ml}$ penicillin, $1 \mathrm{mg} / \mathrm{ml}$ streptomycin, and $2 \mathrm{mM}$ L-glutamine. Human colon biopsies were performed as reported previously $(6,15)$. Treatments of cells with drugs affecting DNA methylation and histone deacetylation were performed as reported previously (11). The medium was replaced every $24 \mathrm{~h}$ with medium containing freshly diluted drugs. At the end of the treatment, the cells were harvested by trypsinization and processed for extraction of total RNA or nuclear protein.

\section{Plasmid DNAs and cell transfections}

The coding sequences of HNF1 $\alpha$, HNF1 $\beta$, Cdx1, and Cdx2 were obtained by PCR, with the reported primer pairs (7), harboring HindIII and $X b a I$ restriction sites, starting with RNA extracted from COLO-205 cells (HNF1 $\alpha)$, MKN-45 cells (HNF1 $\beta$ ), and commercially available (Stratagene 540009; Agilent Technologies Italia, Milan, Italy) human normal mucosa RNA (Cdx1 and Cdx2), using the reported procedure (15). Amplified fragments were restriction digested, cloned into the corresponding sites of the pcDNA3 vector, and sequenced. Because we obtained the variants P130R (16) and S291P (17) of Cdx1 and Cdx2, respectively, from our source, we mutated the plasmids with the QuikChange mutagenesis kit (Agilent Technologies) to obtain the wild-type sequences, according to the manufacturer's protocol.

For permanent transfection, $3 \times 10^{6}$ MDA-MB-231 cells were seeded in $100-\mathrm{mm}$ plates $24 \mathrm{~h}$ in advance, and $20 \mu \mathrm{g}$ of Scal-linearized pcDNA3-HNF1 $\alpha$, pcDNA3-HNF1 $\beta$, or pcDNA3 alone was mixed with $1 \mu \mathrm{g}$ of Xhol-linearized pLKO-puro empty vector (Sigma-Aldrich Italia, Milan, Italy) bearing the puromycin-resistance gene. Transfection solutions were prepared by diluting the linearized DNA with $2 \mathrm{ml}$ of serum-free medium and then adding $2 \mathrm{ml}$ of serum-free medium containing $60 \mu$ l Lipofectamine 2000 (Life Technologies Italia, Monza, Italy). Liposomes were allowed to form for $20 \mathrm{~min}$ at room temperature. The cells were washed twice with serumfree medium, fed with transfection solution, and incubated under regular growth conditions for 3-4 h. At the end, $8 \mathrm{ml}$ of standard complete medium was added, and the incubation was continued. At $2 \mathrm{~d}$ after transfection, the cells were trypsinized and divided into multiple plates. Selection started after an additional $24 \mathrm{~h}$ in the presence of $1 \mu \mathrm{g} / \mathrm{ml}$ puromycin. Resistant colonies were collected, grown, and harvested to extract nuclear protein and total RNA from the same cell suspensions.

For transient transfection, $4 \times 10^{6}$ HEK-293T cells were seeded in $60-\mathrm{mm}$ plates $24 \mathrm{~h}$ in advance, and $5 \mu \mathrm{g}$ of pcDNA3-Cdx1 or pcDNA3-Cdx2 was transfected in $1.5 \mathrm{ml}$ of transfection solutions prepared as above for permanent transfection. The next day, the cells were trypsinized and placed in 
$100-\mathrm{mm}$ plates in the presence of $2 \mathrm{mg} / \mathrm{ml} \mathrm{G} 418$. After an additional $5 \mathrm{~d}$ (changing medium twice), resistant cells were harvested and used for nuclear protein extraction.

For gene silencing, we used Human Mission shRNA Plasmid DNA (Sigma-Aldrich Italia), based on the $\mathrm{pLKO}$ vector, clone TRCN0000017509NM_000458.1-800s1c1TRC 1 (targeting HNF1 $\beta$ ), clone TRCN0000017194NM_000545.3-1039s1c1TRC 1 (targeting $\mathrm{HNF} 1 \alpha$ ), or the pLKO vector alone. They were linearized with BstEII (to destroy the puromycin-resistance gene) and mixed with 1:20 of the same plasmid linearized with ScaI. Transfection of the MKN-45 and MDA-MB-361 cells and puromycin selection were carried out as for the MDAMB-231 cells. Because we found in preliminary experiments that gene silencing was lost after prolonged cell replications (over $4 \mathrm{wk}$ ), we allowed individual or pooled colonies obtained on selection to grow for only $3 \mathrm{wk}$, washed the obtained cell pellets $\left(\sim 0.5-1.0 \times 10^{6}\right.$ cells $)$ with sterile PBS, and divided them into 3 fractions: the first $\left(0.1-0.2 \times 10^{6}\right.$ cells) lysed for RNA extraction, the second $\left(0.4-0.6 \times 10^{6}\right.$ cells) mixed with protease inhibitor cocktail HALT (Thermo Scientific, Waltham, MA, USA) for direct Western blot analysis, and the third plated again for further growth. Sequences were analyzed through the EMBOSS Cpgplot software [European Molecular Biology Laboratory (EMBL), Heidelberg, Germany].

\section{Western blot analysis}

Freshly collected cell pellets were processed to obtain nuclear extracts, with a commercially available kit (NE-PER; Thermo Scientific; ref. 11). Frozen biopsies from the colon were Dounce homogenized and subjected to nuclear extraction as for the cell pellets. Aliquots of nuclear extracts $(5-10 \mu \mathrm{g}$ of protein) were separated by $10 \%$ SDS-PAGE; transferred to a nitrocellulose membrane (Trans-Blot SD Semi-Dry Transfer Cell; Bio-Rad Laboratories S.r.l., Milan, Italy); and blotted with rabbit polyclonal anti-HNF1 $\alpha / \beta$ (sc-8986, 1:200; Santa Cruz Biotechnology Italia, Milan, Italy), rabbit polyclonal anti-H3 (D2B12 4620, 1:500; Cell Signaling Technology, Danvers, MA, USA), rabbit polyclonal anti-Cdx1 (PAB4713, 1:200; Abnova, Taipei, Taiwan), or mouse monoclonal antiCdx2 (M01, 1:200; Abnova) antibodies, according to our published protocol (18).

\section{Competitive RT-PCR}

Quantification of transcripts was performed by competitive RT-PCR, which has been found to be effective for distinguishing different $5^{\prime}$-UTRs without underestimating splice variants (10). Total RNA, prepared and DNase treated with a commercially available kit (SV total RNA isolation system or ReliaPrep cell RNA miniprep; both from Promega Italia, Milan, Italy), was quantitated by fluorometry with Qubit (Life Technologies Italia) and reverse transcribed (11). cDNAs were amplified in a volume of $25 \mu \mathrm{l}$ in the presence of the indicated amounts of competitors (11), for 35 cycles (B3GALT5 LTR, HNF1 $\alpha$, and HNF1 $\beta$ ) or 5 pg competitor for 25 cycles ( $\beta$-actin). The amplification program included a single treatment at $94^{\circ} \mathrm{C}$ for 3 min followed by cycles consisting of $1 \mathrm{~min}$ at $94^{\circ} \mathrm{C}$ (melting), $3.5 \mathrm{~min}$ at $72^{\circ} \mathrm{C}$ (annealing plus extension), and a final extension step at $72^{\circ} \mathrm{C}$ for $8 \mathrm{~min}$ (B3GALT5 and $\beta$-actin) or $1 \mathrm{~min}$ at $94^{\circ} \mathrm{C}$ (melting), $1 \mathrm{~min}$ at $66^{\circ} \mathrm{C}$ (annealing), $2.5 \mathrm{~min}$ at $72^{\circ} \mathrm{C}$ (extension), and a final extension step at $72^{\circ} \mathrm{C}$ for $8 \mathrm{~min}$ (HNF1 $\alpha$ and $-\beta$ ). B3GALT5 LTR and $\beta$-actin competitors and primers were identical with those already reported $(6,10)$. The HNF1 $\beta$ competitor was prepared by subcloning the coding sequence in the pCDM8 vector, digesting with the restriction enzymes $B s p \mathrm{HI}$ and $M s c \mathrm{I}$, and religating. The HNF1 $\beta$ primers were $5^{\prime}$-CAGAGCCATGGGCCTGGGCAGTC-3' (sense) and 5'-CTGCTGGGCCATGTGGCTGCCTG-3' (antisense). The HNF1 $\alpha$ competitor was prepared by digesting pcDNA3-HNF1 $\alpha$ with the restriction enzymes XhoI and Bsp1407I, blunting with Klenow, and religating. The HNF1 $\alpha$ primers were 5'-GCCATGGTTTCTAAACTGAGCCAG-3' (sense) and 5'-GTCCATAGCGCACACCGTGGAC-3' (antisense).

Parallel PCR amplifications were performed on known amounts of standard cDNAs premixed with the competitors. Standard cDNAs were the original cloned sequences quantitated and diluted as for the competitors. Aliquots of PCR reactions were analyzed on $1 \%$ agarose gels stained with ethidium bromide. Quantification was performed by densitometric scanning of the gel, and the amounts of amplified target $\mathrm{cDNAs}$ were calculated from their respective standard curves and normalized by that for $\beta$-actin. The target:competitor ratios had been shown to make the PCR results quantitative (19).

\section{Luciferase assay}

The DNA fragment containing the sequence from -148 to -128 from the LTR transcription initiation site, cloned in the pGL3 vector as reported previously (10), was used as the LTR promoter (pGL3-LTR).

For transfection, 50,000-80,000 host cells were plated $20 \mathrm{~h}$ in advance in 96-well plates with $0.1 \mathrm{ml}$ culture medium. Transfection solutions were prepared by mixing $200 \mathrm{ng}$ of test DNA (pGL3-LTR alone or $100 \mathrm{ng}$ pGL3-LTR plus $100 \mathrm{ng}$ pcDNA3-based plasmids) with $10 \mathrm{ng}$ of the Renilla luciferase expression vector pRL-CMV (Promega Italia) in $25 \mu \mathrm{l}$ serumfree medium. Serum-free medium $(25 \mu \mathrm{l})$ containing $0.6 \mu \mathrm{l}$ Lipofectamine 2000 was then added to the DNA dilution, and the complete solution was used as above for all other transfections. After the addition of $0.1 \mathrm{ml}$ standard medium, the incubation was continued for $20 \mathrm{~h}$. The cells were then washed, lysed, and assayed for luciferase activity (10).

\section{EMSA}

EMSAs were performed with the Lightshift chemiluminescence kit (Thermo Scientific) according to the manufacturer's recommendations, but the binding reaction volume was scaled down to $10 \mu \mathrm{l}$. DNA probes biotinylated at the 3 ' end were synthesized by Eurofins-MWG Operon (Ebersberg, Germany) and diluted to $1 \mathrm{nM}$ in the assay. Nuclear extracts were prepared as for Western blot analysis, and $1 \mu \mathrm{l}$ of extract (3-4 $\mu \mathrm{g}$ of protein) was added to each binding reaction. AntiHNF-1 antibody was incubated for $30 \mathrm{~min}$ on ice in the binding reaction before the probes were added. Cdx competitor DNA was the 24-bp SIF1 sequence 5'-GGGTGCAATAAAACTTTATGAGTA-3' deduced from Suh et al. (20). DNA/ protein complexes were separated by $5 \%$ native PAGE, transferred to a nylon membrane, cross-linked under UV light, and visualized as reported (10).

\section{RESULTS}

\section{Detection of $\mathrm{HNF} 1 \alpha / \beta$ and $\mathrm{Cdx} 1 / 2$ in colon cancer biopsies and cell lines expressing different amounts of B3GALT5 LTR transcript}

In preliminary experiments, we determined the expression of the B3GALT5 LTR transcript in matched pairs of biopsies representing colon cancers and surround- 
ing normal mucosae. As expected, owing to the strong prevalence of the transcript in these tissues, the results were similar to those that had been obtained measuring the B3GALT5 coding sequence $(6,7)$, which is common to all transcripts. Expression levels, in fact, ranged from 11 to $1 \mathrm{fg} / \mathrm{pg}$ of $\beta$-actin (average, 3.9) and from $0.5 \mathrm{fg} / \mathrm{pg}$ of $\beta$-actin to undetectable levels (average, 0.13 ), in normal colon mucosae and colon cancers, respectively (Supplemental Fig. S1A). In relation to levels in the matched pairs, the amount of LTR transcript decreased in cancer from $4-$ to $>200$-fold. To assess the relationship between the LTR transcript and the candidate transcription factors $\mathrm{HNF} 1 \alpha / \beta$ and $\mathrm{Cdx} 1 / 2$, we determined their levels in nuclear protein extracted from representative biopsies. We had enough material available for extracting nuclear protein from 8 cancer biopsies, but from only 2 normal mucosa specimens, and we therefore first compared the cancer samples with various cell lines presenting a wide range of expression of the LTR transcript. We were surprised to find that, in all cancer samples tested, HNF1 $\alpha$ and HNF1 $\beta$ were easily detected, whereas Cdx1 and Cdx2 were almost undetectable, irrespective of the amount of LTR transcript expressed (Fig. 1). We also found that the COLO-205 and SW-1116 cells, which expressed the highest amount of LTR transcript $(\sim 15$ and $6 \mathrm{fg} / \mathrm{pg}$ of $\beta$-actin, respectively), expressed only $\mathrm{HNF} 1 \alpha$, at levels similar to those in samples expressing low or no LTR transcript, such as the hepatoma cell lines Huh-7 and Hep-3B. The MKN-45 and HT-29 cells expressed both HNF $1 \alpha$ and HNF1 $\beta$, but the transcript levels were lower than in the COLO-205 or SW-1116 cells. In contrast, the MDA-MB-361 cells expressed only HNF1 $\beta$ and slightly more LTR transcript than did the MKN-45 or HT-29 cells. Of interest, the cells lacking HNF1 $\alpha / \beta$ also lacked LTR transcript, but not vice versa. Cdx1 and Cdx2 remained undetectable under reference conditions in all tested samples. A weak Cdx2 spot became detectable after a longer exposure in the HCT-15 cells and in two cancer biopsies expressing no LTR tran-
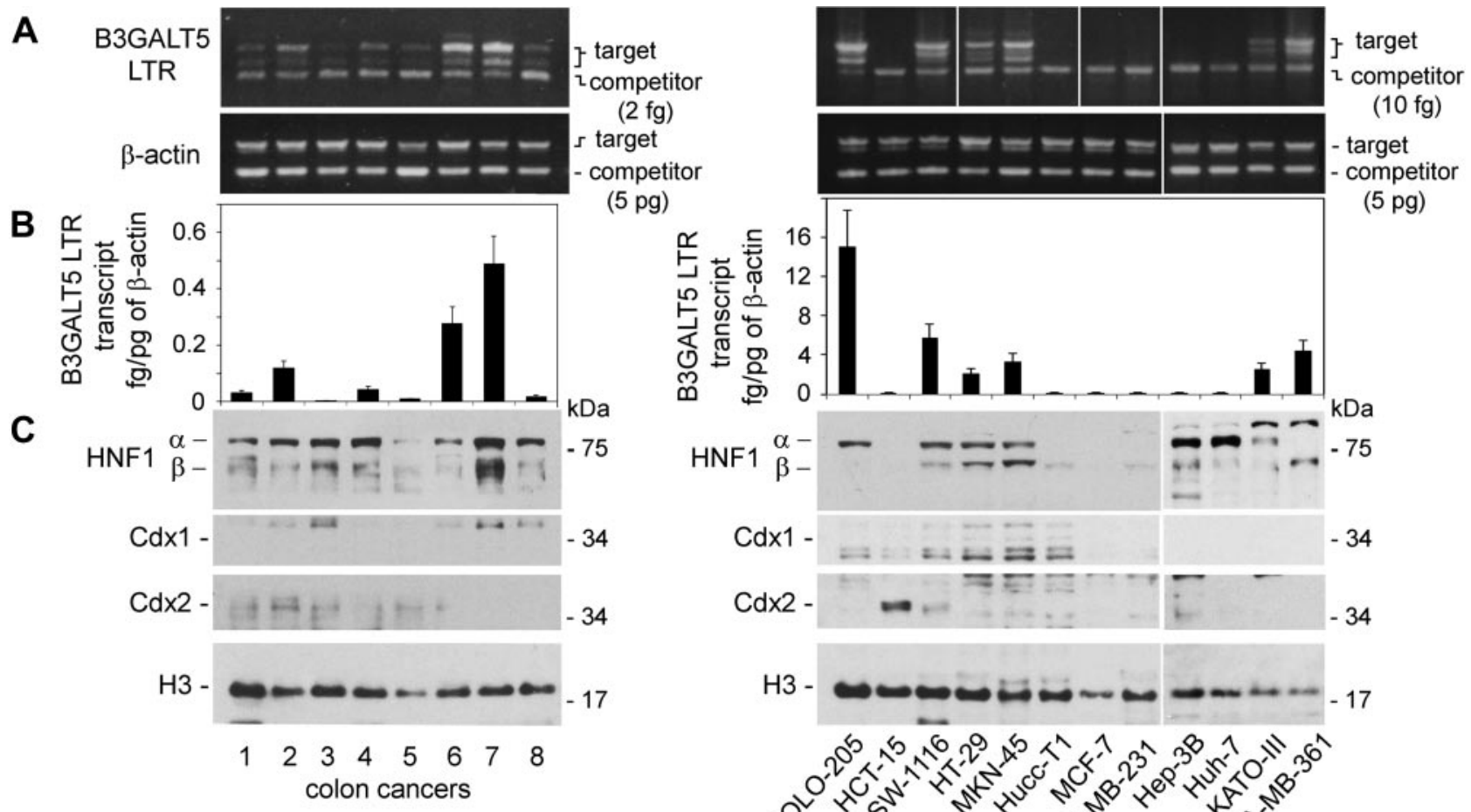

$(5 \mathrm{pg})$

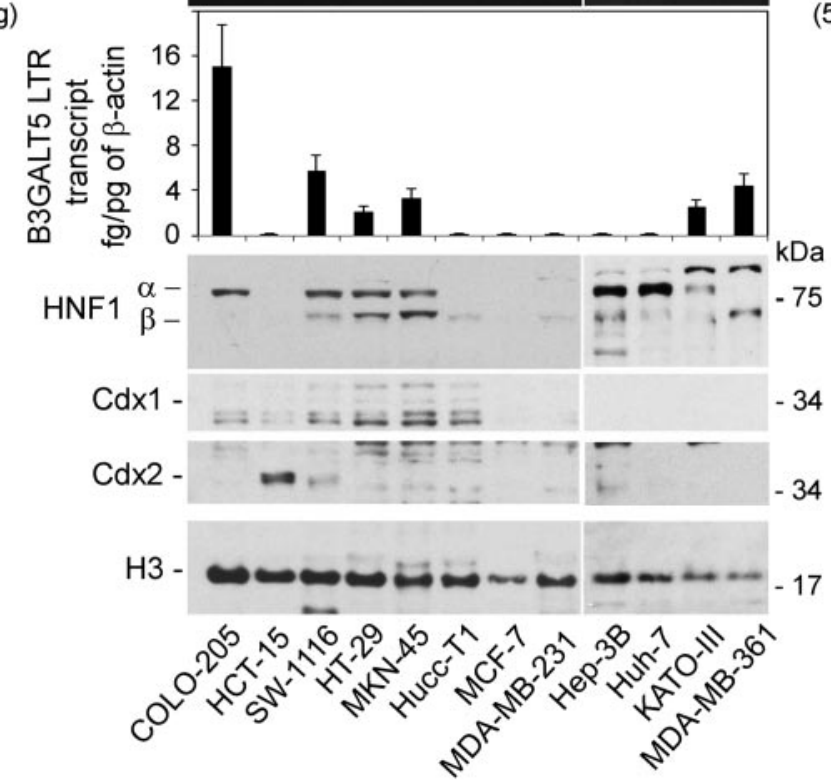

$(5 \mathrm{pg})$

Figure 1. Detection of transcription factors $H N F 1 \alpha / \beta$ and $\operatorname{Cdx} 1 / 2$ in human colon cancers and cell lines expressing various amounts of B3GALT5 LTR transcript. A) RNA extracted from cancers and cell lines was reverse transcribed, and normalized amounts of the resultant first-strand cDNAs were mixed with the indicated amounts of competitor (truncated) cDNAs and subjected to PCR (25 and 35 cycles for $\beta$-actin and B3GALT5 LTR, respectively), using primers specific to $\beta$-actin and B3GALT5 LTR transcript, respectively. Note the different amounts of B3GALT5 LTR competitor cDNA used in the case of colon cancers, cell lines (present figure), and matched pairs of colon biopsies (Supplemental Fig. S1A). Twenty percent of each amplification reaction was electrophoresed in a $1 \%$ agarose gel and visualized by ethidium bromide staining. The target doublet corresponded to the alternative splicing that had been reported (10). Representative gels are shown. Samples were not all run on the same gel, as indicated by the vertical white spaces between the gels. $B$ ) Densitometric scanning of gel images was performed to quantitate the amounts of B3GALT5 LTR transcript, which were calculated from a standard curve and normalized to the amounts calculated for $\beta$-actin. Results are means \pm SD of 3 determinations. Note the different scales used for colon cancer and the cell lines. C) Nuclear extracts (5-10 $\mu$ g of protein) were separated by $10 \%$ SDS-PAGE and transferred to a nitrocellulose membrane that was blotted with anti-HNF1 antibody (recognizing both HNF1 $\alpha$ and HNF1 $\beta$ ), or anti-Cdx1, anti-Cdx2, or anti-histone H3 antibodies, followed by an HRP-labeled secondary antibody and chemiluminescence detection. Longer exposures were necessary to detect a visible spot for Cdx1 and Cdx2. For comparative quantitation, see Supplemental Fig. S1B. 
script at all. To assess the sensitivity of the anti-Cdx antibodies, we analyzed the nuclear protein extracted from the HEK-293T cells transfected with Cdx1 or Cdx2 cDNAs and found very strong signals under the conditions used for detection (Supplemental Fig. S1B). Finally, in the two available normal colon mucosa biopsies (Supplemental Fig. S1C), we detected HNF1 $\alpha / \beta$ only. Their levels were not related to those of B3GALT5 LTR transcript, as found in the cancer cell lines.

\section{Detection of B3GALT5 LTR transcript in cell models expressing various amounts of HNF1 $\alpha$ or HNF1 $\beta$}

To better assess the role of $\mathrm{HNF} 1 \alpha / \beta$, we planned to overexpress the factors in a suitable cell model. In preliminary experiments, we used Western blot analysis to determine the expression levels of $\mathrm{HNF} 1 \alpha / \beta$ transcripts in cell lines that do not express the protein, such as HCT-15 and Hucc-T1 (Fig. 1). Both lines unexpectedly expressed HNF1 $\beta$ transcript at high levels. We therefore extended the analysis to many other cell lines and found that HNF1 $\beta$ RNA was commonly expressed, even in cells where the protein was undetectable by Western blot analysis (Supplemental Fig. S2), suggesting a crucial post-transcriptional regulation of the gene. This phenomenon was not evident in the case of HNF1 $\alpha$ (Supplemental Fig. S2). The breast cancer cell line MDA-MB-231, lacking both HNF1 $\alpha$ and HNF1 $\beta$ transcripts, was thus chosen for the transfection experiments.

The clones, analyzed by Western blot analysis (Fig. 2A, bottom panels), were found to express various, but rather high, levels of $\mathrm{HNF} 1 \alpha$, compared with that in the Huh-7 cells, and various but low levels of HNF1 $\beta$, compared with that in the MDA-MB-361 cells. B3GALT5 LTR transcript became detectable, although at low levels, in all the clones expressing either HNF1 $\alpha$ or HNF1 $\beta$ (Fig. $2 A$, top panel). The amount of LTR transcript, in fact, ranged from 0.05 to $0.5 \mathrm{fg} / \mathrm{pg}$ of $\beta$-actin. However, even weak expression of HNF1 $\beta$ induced transcription of LTR mRNA. In particular, the clones $\beta-1$ and $\alpha-4$ expressed similar levels of LTR transcript, whereas the amount of HNF1 $\beta$ in the former was much lower than that of HNF $1 \alpha$ in the latter (Fig. 2A). Notwithstanding the low levels of HNF1 $\beta$ protein in the clones, the corresponding amount of transcript was very high, the highest detected among the tested cells (Supplemental Fig. S2), confirming that regulation of the HNF1 $\beta$ gene is largely post-transcriptional. To focus on the role of HNF1 $\beta$, we silenced HNF1 $\alpha$ in the MKN-45 cells, which express both factors, and HNF1 $\beta$ in the MDA-MB-361 cells, which express HNF1 $\beta$ only. Using plasmids encoding shRNAs targeting either factor, we obtained a transient but strong reduction of expression of HNF1 $\alpha$

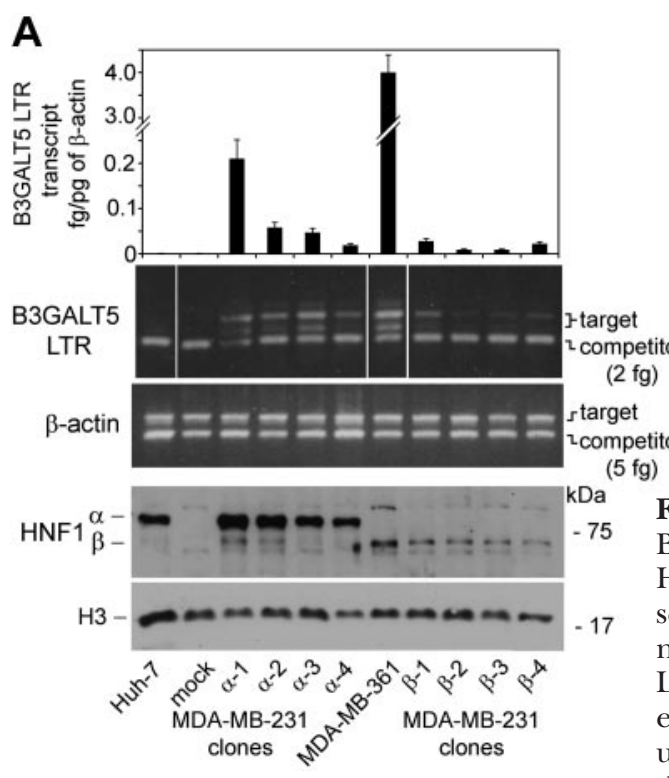

transcript, and MDA-MB-361 cells, expressing references. $B$ ) MKN-45 cells, expressing both HNF1 $\alpha$ and HNF1 $\beta$, as well as B3GALT5 LTR transcript, were transfected with the pLKO plasmid bearing an shRNA sequence specifically targeting HNF1 $\alpha$. Individual clones resistant to puromycin selection were allowed to grow for $3 \mathrm{wk}$, and the cells obtained were used for Western blot analysis, RNA extraction, or replating for further growth up to $5 \mathrm{wk}$. A control clone was prepared in parallel transfecting cells with the empty pLKO vector. Western blot analysis (top panel) and competitive RT-PCR analyses (bottom panels) were then performed as reported in Fig. 1. Detection of HNF1 $\beta$ was used as internal reference to evaluate HNF1 $\alpha$ silencing and re-expression. $C$ ) MDA-MB-361 cells, expressing HNF1 $\beta$ only, as well as B3GALT5 LTR transcript, were transfected with pLKO plasmid bearing an shRNA sequence that specifically targets HNF1 $\beta$. Individual or pooled clones resistant to puromycin selection were allowed to grow for $3 \mathrm{wk}$, and the obtained cells were used for Western blot analysis, RNA extraction, or replating for further growth up to $5 \mathrm{wk}$. A control clone was prepared in parallel, transfecting cells with the empty pLKO vector. Western blot analysis (top panel) and competitive RT-PCR analyses (bottom panels) were then performed as reported in Fig. 1. Detection of histone H3 was used as internal reference to evaluate HNF1 $\beta$ silencing and reexpression. 


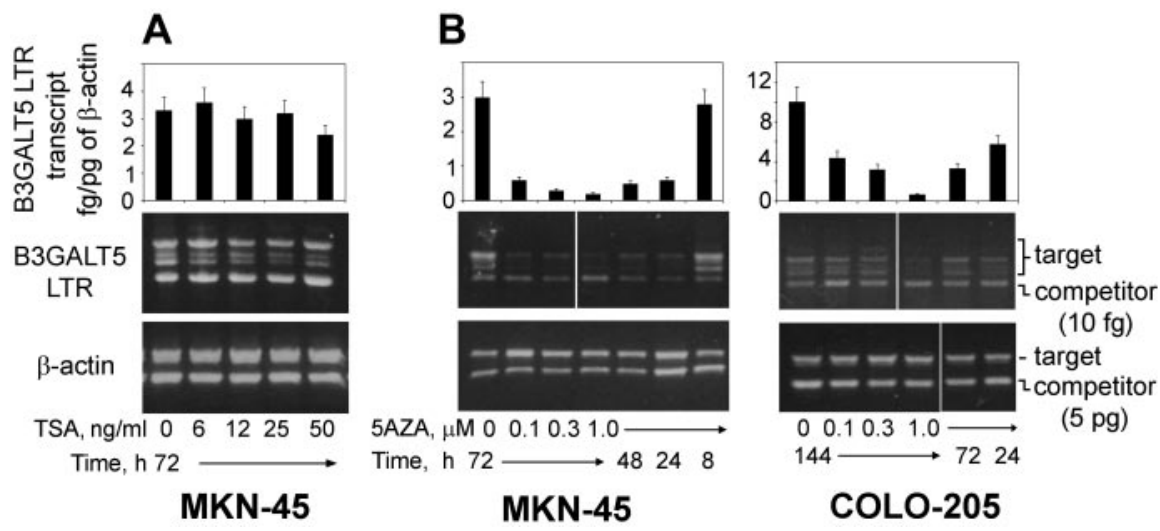

\section{C}

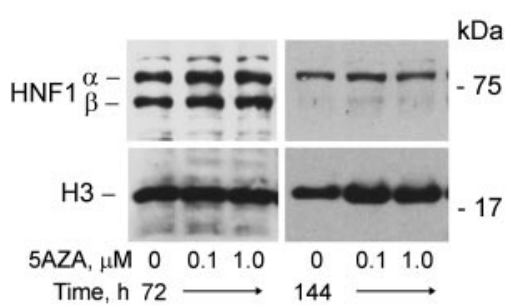

MKN-45 COLO-205

Figure 3. Effect of TSA and 5AZA treatment on the expression of B3GALT5 LTR transcript in cultured cells. A) MKN-45 cells were treated with various amounts of the histone deacetylase inhibitor TSA. B) MKN-45 and COLO-205 cells were treated with various amounts of the DNA methyltransferase inhibitor 5AZA for different times. In both instances, detection and quantification of B3GALT5 LTR transcript by competitive RT-PCR were performed as in Fig. 1. Treatments were performed in duplicate. At the end of the treatments, cells were harvested, washed with PBS, and processed to extract RNA and nuclear protein from the same cell suspension. Results are means \pm SD of 2 determinations performed on each duplicate. C) Nuclear protein extracted from representative data points were subjected to Western blot analysis, as reported in Fig. 1.

in the MKN-45 cells (Fig. 2B, top panel) and of HNF1 $\beta$ in the MDA-MB-361 cells (Fig. 2C, top panel). The former had no effect on LTR transcript levels (Fig. 2B, bottom panel), but the latter was accompanied by a dramatic reduction of the transcript (Fig. 2C, bottom panel). After longer periods following transfection, recovery of HNF1 $\alpha$ expression in the MKN-45 cells did not affect the LTR transcript levels, whereas recovery of HNF1 $\beta$ expression in the MDA-MB-361 cells restored the amount of the transcript. These data indicate that HNF $1 \alpha$ and HNF1 $\beta$ play an interchangeable, but not a cumulative, role, and suggest that, although necessary for B3GALT5 transcription, they are not sufficient to explain transcript modulation.

To remove potential epigenetic constraints, we performed trichostatin A (TSA) and 5AZA treatments, starting with the MKN-45 cells, given that they were found to be sensitive to such treatments in the case of the native B3GALT5 transcript (11). We found that TSA treatment (Fig. 3A) had no effect on the LTR transcript, whereas, surprisingly, 5AZA strongly impaired expression (Fig. $3 B$ ). We repeated the treatment on the COLO-205 cells, because they express LTR transcript at the highest levels found, and obtained similar results (Fig. 3B). In fact, even in these cells, 5AZA treatment impaired LTR expression in a doseand time-dependent manner. In both treated cell lines, B3GALT5 LTR transcript dropped down to the levels measured in some colon cancer biopsies or HNF $1 \alpha / \beta$ - transfected MDA-MB-231 clones, whereas the levels of HNF1 proteins remained almost unaffected (Fig. 3C). Because the 650-bp LTR transposon contains only 7 dispersed CG pairs, and no CpG island is present in the proximal sequences (Fig. 4), this result suggests that the regulatory effect of methylation resides outside and far from the LTR promoter.

\section{In vitro evaluation of the B3GALT5 promoter}

To confirm the hypothesis that the B3GALT5 LTR promoter, although necessary, is unable to regulate transcription per se, we performed luciferase assays with a reporter plasmid, in which luciferase is placed under the control of the LTR sequence, and EMSAs, with the LTR sequence used as a probe. Taking advantage of the availability of cell lines and clones showing various combinations of the expression levels of the B3GALT5 LTR transcript and HNF $1 \alpha / \beta$ proteins, we used them as the host cells for transfecting the LTR/luciferase plasmid or as the sources for extracting nuclear protein to be tested with the LTR probe by EMSA.

As shown in Fig. 5A, by luciferase reporter assay, the highest activity was found in the MKN-45 cells, which expressed high levels of both HNF1 $\alpha$ and HNF1 $\beta$ and moderate levels of LTR transcript, followed by the Huh-7 cells and MDA-MB-231 clone $\alpha-1$, which expressed high levels of HNF1 $\alpha$ only and no or low LTR transcripts. Conversely, much lower luciferase activity

CpG
islands
(length, bp)

Figure 4. CpG islands detected in the context of the B3GALT5 gene. The genomic sequence of chromosome 21 (0.26 Mbp) encompassing $B 3 G A L T 5$ and intergenic regions was analyzed with EMBOSS Cpgplot software (EMBL), with the following parameters: observed:expected ratio $>0.6, \mathrm{C}+\mathrm{G}>50 \%$, and minimum length, 120 bp. One CpG island was present near exon

1A, which regulates the cognate promoter (11). Several shorter stretches of CpG dinucleotides were instead detectable in the intergenic regions. 
Figure 5. Luciferase activity assay of the B3GALT5 LTR promoter. The genomic sequence -148 to -128 , calculated from the transcription initiation site of B3GALT5 LTR mRNA, encompassing part of the LTR transposon, was cloned in the pGl3 vector carrying the firefly luciferase gene (pGl3-LTR) and transfected together with a Renilla luciferase reporter expression plasmid in various host cells. A) Host cells were from cell lines or clones that express the different amounts of HNF $1 \alpha / \beta$ and B3GALT5 LTR transcript shown in Figs. 1 and 2. The pGL3-control vector, bearing the SV40 promoter, and the pGl3-basic vector, lacking any promoter sequence, were also transfected for normalization and control. Firefly luciferase activity was measured $24 \mathrm{~h}$ later, calculated relative to the Renilla luciferase activity determined for each sample, and expressed as a fraction of the activity measured with the pGL3-control in the same host cells. $B$ ) pGL3-LTR and Renilla luciferase plasmids were mixed with expression plasmids having the coding region of transcription factors cloned in the pcDNA3 vector, which bears the SV40 origin of replication, and were transfected into HEK-293T cells, which are able to replicate plasmids with the SV40 origin of replication. Firefly luciferase activity was measured $24 \mathrm{~h}$ later and expressed relative to the Renilla luciferase activity determined for each sample. Transfection with pGL3-LTR and Renilla luciferase plasmids mixed with pcDNA3 vector alone was performed as the control. Results are expressed as means \pm sD of 2 experiments performed in triplicate.

was detected in the SW-1116 cells, expressing high levels of transcript and moderate levels of HNF1 $\alpha$. In the MDA-MB-361 cells expressing moderate levels of transcript but low HNF1 $\beta$ alone, luciferase activity was minimal, and almost undetectable in the MDA-MB-231 clone $\beta-1$, which expresses minimal amounts of both LTR transcript and HNF1 $\beta$. We also transfected HEK293T cells, which lack expression of $\mathrm{HNF} 1 \alpha / \beta$ and Cdx1/2 and can replicate plasmids with the SV40 origin of replication (pcDNA3), in the presence of transcription factor cDNAs cloned in the pcDNA3 vector. The addition of each plasmid coding $H N F 1 \alpha / \beta$ or Cdx1/2 enhanced luciferase activity (Fig. $5 B$ ), with a preference for HNF $1 \alpha$ followed by HNF1 $\beta$ and Cdx2, whereas Cdx1 was less effective.

By EMSA, we found that the LTR sequence, when used as a probe, formed 1 specific complex plus 1 or 2 other nonspecific complexes (Fig. 6). The single specific complex was the most retarded and appeared as a doublet, corresponding to HNF $1 \alpha / \beta$ binding. In fact, it was found only with nuclear protein extracted from cell lines expressing HNF1 $\alpha / \beta$, was affected by the antiHNF1 antibody, and was outcompeted by an excess of the unlabeled probe sequence (Fig. 6A). The complex was also evident with nuclear proteins extracted from cells expressing very low to undetectable levels of LTR transcript but very high levels of HNF1 $\alpha$, such as the Huh-7, Hep-3B, and MDA-MB-231 clones $\alpha-1$ or $\alpha-3$. The complex was detectable but much less evident with the protein extracted from the MDA-MB-361 cells, which expressed moderate levels of LTR transcript and low levels of HNF1 $\beta$, and was undetectable with the MDA-MB-231 clone $\beta-1$, which expressed very low levels of both. The other complexes were not outcompeted by the unlabeled probe and should be considered nonspecific (Fig. 6B). In particular, the complex that migrated as the less retarded doublet was formed by almost any nuclear extract, including those prepared from cells not expressing B3GALT5 LTR and unable to drive luciferase activity on transfection with the LTR construct. It migrated much faster than true $\mathrm{Cdx} 1$ or Cdx2 complexes formed by the same LTR probe with authentic $\mathrm{Cdx} 1$ or $\mathrm{Cdx} 2$ protein and was not competed out by the SIF1 sequence, as occurred with the true $\mathrm{Cdx} 1$ or Cdx2 complexes formed by recombinant factors (Fig. 6C). Although the LTR promoter bound $\mathrm{Cdx} 1 / 2$ in vitro, taken together, the data indicate that the HNF1 binding site is the only functional sequence brought about by the insertion of the LTR transposon.

\section{DISCUSSION}

We observed that HNF $1 \alpha$ and HNF1 $\beta$ are necessary to activate the B3GALT5 LTR promoter, playing an interchangeable and not cumulative role, but they are unable to modulate transcription, which instead depends on the distal regulatory elements that are active when methylated and are unknown at present.

The complex role played by HNF1 was defined by several lines of evidence. First of all, in any cell line lacking both HNF1 $\alpha$ and HNF1 $\beta$, the B3GALT5 LTR transcript was always undetectable; moreover, transfection of one of these cells with either HNF $1 \alpha$ or HNF1 $\beta$ cDNA induced LTR transcription. In addition, shRNAmediated silencing of HNF1 $\beta$ in cells expressing only HNF1 $\beta$ strongly impaired LTR expression. These results clearly indicate that HNF1 is necessary for transcription. Because the transcript was detected in cell lines and clones expressing either HNF $1 \alpha$ or $H N F 1 \beta$ alone, and shRNA-mediated silencing of HNF1 $\alpha$ in cells expressing both HNF1 $\alpha$ and HNF1 $\beta$ does not affect LTR expression, the two forms appear to be not cooperative, but interchangeable, as reported in the gut (21), but not in the kidney (22). On the other hand, the expression levels of either HNF1 form, or of both forms when expressed together, cannot explain the wide expression range of the LTR transcript. In 

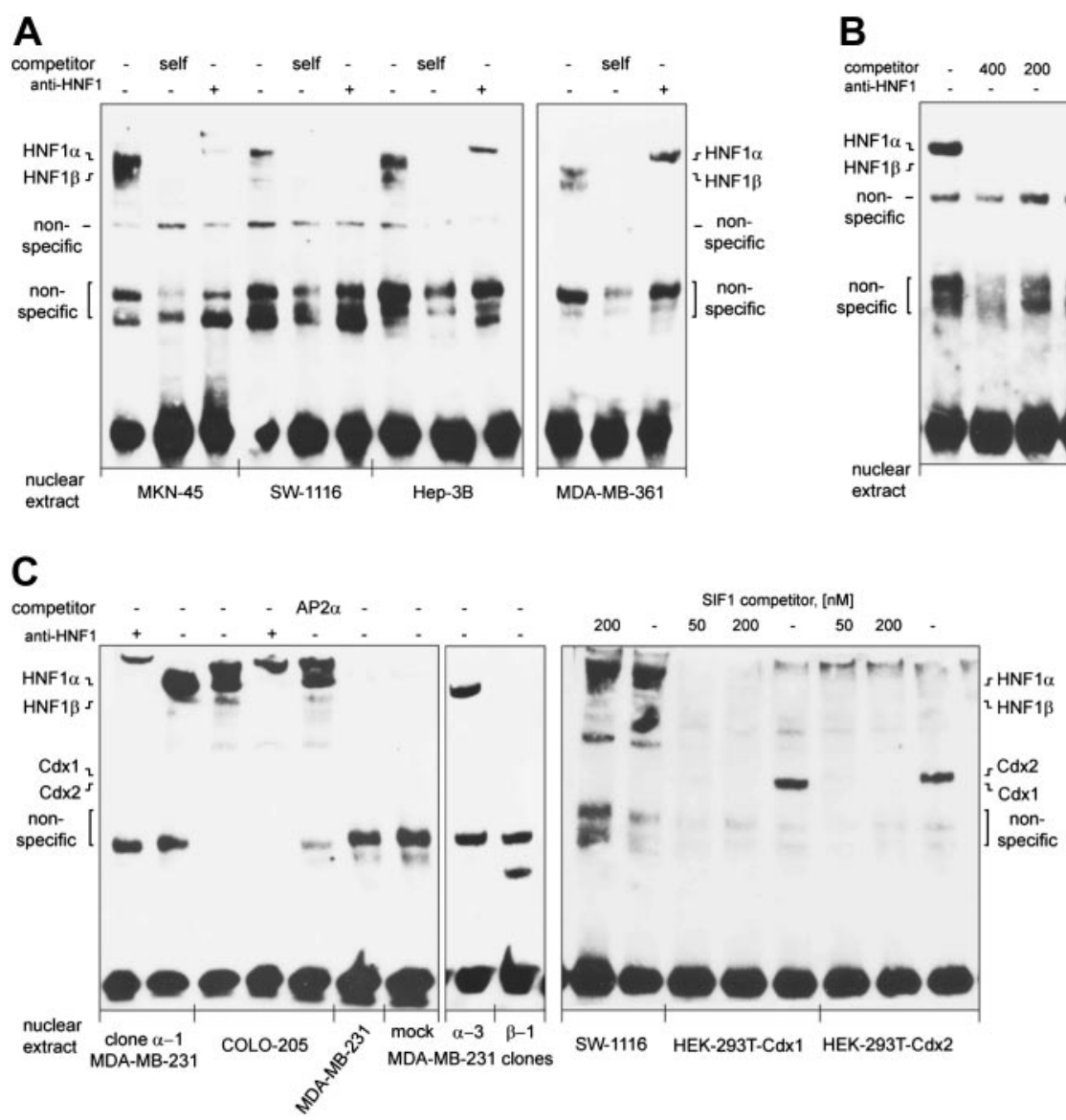

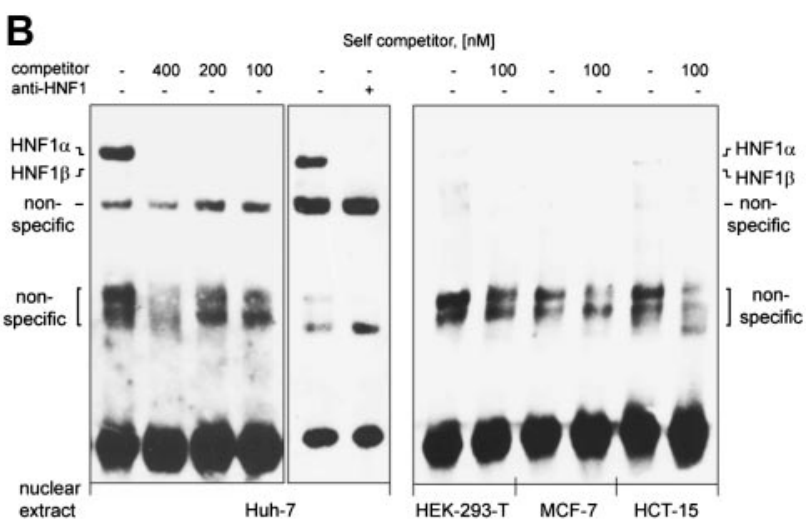

Figure 6. Characterization of the binding properties of the B3GALT5 LTR promoter. EMSAs were performed by incubating a biotinylated oligonucleotide probe (nt -151 to -112 , calculated from the transcription initiation site of B3GALT5 LTR mRNA) with nuclear protein extracted from cell lines or clones expressing the different amounts of HNF $1 \alpha / \beta$ and B3GALT5 LTR transcripts shown in Figs. 1 and 2, as well as from HEK-293T cells expressing recombinant Cdx1 or Cdx2 (Supplemental Fig. S1B). Competitor oligonucleotides were the unlabeled oligonucleotide probe (self), the consensus sequence of AP2 $\alpha$ (5'-GATCGAACTGACCGCCCGCGGCCCGT-3') used as an irrelevant DNA control, and the consensus sequence of Cdx1/2 deduced from the sucrase-isomaltase promoter (SIF1) reported by Suh et al. (20). They were present in 100-fold molar excess in the incubation mixture, unless differently indicated. The resultant complexes were separated by $5 \%$ native PAGE and visualized by chemiluminescence. $A$ ) MKN-45, SW-1116, and MDA-MB-361 cells expressed the B3GALT5 LTR transcript, whereas Hep-3B cells did not. B) No cell line expressed the B3GALT5 LTR transcript. C) MDA-MB-231 cells did not express the B3GALT5 LTR transcript, whereas clones expressed low levels of the transcript, and COLO-205 and SW-1116 cells expressed high levels. fact, comparable amounts of HNF1 were detected in the COLO-205 or SW-1116 cells, which expressed the highest levels of LTR transcript, and in the cells, clones, or colon cancer biopsies, which expressed 10- or 100fold less, or even undetectable, LTR transcript. This evidence prompts the question of what other factor or mechanism affects B3GALT5 LTR transcription and is responsible for cancer down-regulation. We found that Cdx1 and Cdx2 were not involved, although they bound and activated the LTR promoter in vitro. In fact, the amounts of $\mathrm{Cdx} 1 / 2$ in cells or tissues expressing the B3GALT5 LTR transcript were negligible, and the few samples expressing detectable amounts of $\mathrm{Cdx} 2$ (HCT-15 cells and colon cancer biopsies 1 and 2) lacked B3GALT5 LTR transcript. Moreover, nuclear protein extracted from cells expressing the transcript never formed Cdx1/2 complexes in the EMSAs, and the less retarded doublet detected by EMSA with the LTR probe and previously proposed to depend on Cdx1/2 binding (7) did not behave as true Cdx1/2 complexes, but was found to be a nonspecific artifact formed by any nuclear protein extract, including those from cells not expressing the transcript at all. Altogether, these data indicate that the HNF1 binding site is the only functional part of the LTR promoter and that no other binding sites for stimulatory or inhibitory factors, including Cdx1/2, are physiologically relevant.

Conversely, DNA demethylation, obtained through 5AZA treatment of cells expressing the B3GALT5 LTR transcript, reproduced in vitro the down-regulation of the transcript observed among cell lines and cancer biopsies in vivo. In fact, in treated cells, the levels of B3GALT5 LTR transcript decreased from 3 or $10 \mathrm{fg} / \mathrm{pg}$ $\beta$-actin to $<0.2 \mathrm{fg} / \mathrm{pg}$, whereas the amounts of HNF1 remained almost unchanged. LTR and proximal sequences do not contain CpG islands, and we therefore conclude that methylation-sensitive DNA sequences represent elements involved in transcriptional regulation residing outside the LTR sequence, probably distant from the promoter. Alignment of the LTR sequence and the whole B3GALT5 gene in the context of chromosome 21 (Fig. 4) revealed a single typical promoter-associated CpG island, already characterized in detail and responsible for regulating transcription of native B3GALT5 mRNA (11). In addition, several very short CpG islands were detected: one in an intron and the others in the intergenic regions. Unfortunately, because of the extremely high homology of this human 
sequence with that of the other primates sharing the LTR transposon, no prediction can be made in silico about the relevance of any of these islands. Alternatively, the region involved could be $>0.1 \mathrm{Mbp}$ away from the LTR sequence.

We thus propose a model of B3GALT5 transcription predicting that a distant DNA region, methylated in normal colon mucosa and cell lines, such as COLO-205 or SW-1116, is demethylated in colon cancer, in several cell lines, and even in other tissues still expressing HNF1. The degree of demethylation down-regulates transcription to complete silencing, as in the Hep-3B and Huh-7 cells in this study.

DNA hypomethylation was indeed the first epigenetic abnormality detected in human cancers $\sim 30 \mathrm{yr}$ ago (23). However, it has received much less attention in the past decade than the opposite modification, hypermethylation, frequently associated with silencing of tumor-suppressor and other genes. Only recently, high-resolution, genome-wide analyses revealed an independent and relevant role of hypomethylation in cancer formation and progression (24, 25). Among various DNA sequences affected by cancer-associated hypomethylation, transcription control elements appeared to be of special interest. In particular, it was found that genes associated with hypermethylated distant control elements are more frequently down-regulated in cancer (26).

No information about the nature, exact location, or mechanism of action of such a distant sequence is available for B3GALT5 transcription at present. It may represent a typical CpG island, or a "shore," as recently proposed (27), or instead include stretches of CpG dinucleotides shorter than the CpG islands associated with promoters, such as those present in the intergenic regions of B3GALT5. Methylation of such stretches of CpG dinucleotides is emerging as a relevant aspect of transcriptional control (28) and is responsible for recruitment of alternative promoters, regulation of noncoding RNA synthesis, and modulation of enhancer activity. In particular, hypomethylation of enhancer sequences is reported to negatively regulate transcription in cancer and during tissue differentiation (28). The occurrence of distal regulatory elements that bind transcription factors in a methylation-dependent manner was recently reported in breast cancer (29). Other types of genome-wide analyses have indicated that HNF1 can play such a role in the kidney (30) and in the liver (31). B3GALT5 transcription thus represents a promising model to address such novel issues, since hypomethylation of distant sequences, acting on the LTR transcript, and promoter hypermethylation, acting on the native transcript (11), cooperate on one gene to obtain full cancer-associated silencing.

Our present findings also contribute to a better understanding of the mechanism of evolutionary stabilization of the LTR transposon in comparison with the models hypothesized for other mobile elements (32). In fact, reported evidence suggests that the transposon is already active at the time of insertion (9) and may take advantage of the analogous sequence present in the type $\mathrm{B}$ promoter, which is much less active because of its opposite orientation (10). We now propose that effective interaction with a distant element plays a crucial stabilizing effect, probably due to the precise localization of insertion, which in turn allows not only stronger HNF1-associated expression, but also the most precise tissue specificity, obtained through the overlapping epigenetic control exerted by the distal element.

A.Z. was supported by the Ph.D. Program in Molecular Medicine of the University of Milan.

\section{REFERENCES}

1. Isshiki, S., Kudo, T., Nishihara, S., Ikehara, Y., Togayachi, A., Furuya, A., Shitara, K., Kubota, T., Watanabe, M., Kitajima, M., and Narimatsu, H. (1999) Cloning, expression, and characterization of a novel UDP-galactose: $\beta$-N-acetylglucosamine $\beta 1,3-$ galactosyltransferase ( $\beta 3 \mathrm{Gal}-\mathrm{T} 5)$ responsible for synthesis of type 1 chain in colorectal and pancreatic epithelia and tumor cells derived therefrom. J. Biol. Chem. 274, 12499-12507

2. Kannagi, R., Izawa, M., Koike, T., Miyazaki, K., and Kimura, N. (2004) Carbohydrate-mediated cell adhesion in cancer metastasis and angiogenesis. Cancer Sci. 95, 377-384

3. Läubli, H., and Borsig, L. (2010) Selectins promote tumor metastasis. Semin. Cancer Biol. 20, 169-177

4. Duffy, M. J., Sturgeon, C., Lamerz, R., Haglund, C., Holubec, V. L., Klapdor, R., Nicolini, A., Topolcan, O., and Heinemann, V. (2010) Tumor markers in pancreatic cancer: a European Group on Tumor Markers (EGTM) status report. Ann. Oncol. 21, 441-447

5. Mare, L., Caretti, A., Albertini, R., and Trinchera, M. (2013) CA19.9 antigen circulating in the serum of colon cancer patients: where is it from? Int. J. Biochem. Cell Biol. 45, 792-797

6. Salvini, R., Bardoni, A., Valli, M., and Trinchera, M. (2001) beta 1,3-Galactosyltransferase beta 3Gal-T5 acts on the GlcNAcbeta $1 \rightarrow 3$ Galbeta $1 \rightarrow 4$ GlcNAcbeta $1 \rightarrow \mathrm{R}$ sugar chains of carcinoembryonic antigen and other N-linked glycoproteins and is downregulated in colon adenocarcinomas. J. Biol. Chem. 276, 35643573

7. Isshiki, S., Togayachi, A., Kudo, T., Nishihara, S., Watanabe, M., Kubota, T., Kitajima, M., Shiraishi, N., Sasaki, K., Andoh, T., and Narimatsu, H. (2003) Lewis type 1 antigen synthase (beta3Gal-T5) is transcriptionally regulated by homeoproteins. J. Biol. Chem. 278, 36611-36620

8. Dunn, C. A., Medstrand, P., and Mager, D. L. (2003) An endogenous retroviral long terminal repeat is the dominant promoter for human beta1,3-galactosyltransferase 5 in the colon. Proc. Natl. Acad. Sci. U. S. A. 100, 12841-12846

9. Dunn, C. A., van de Lagemaat, L. N., Baillie, G. J., and Mager, D. L. (2005) Endogenous retrovirus long terminal repeats as ready-to-use mobile promoters: the case of primate beta3GAL-T5. Gene $\mathbf{3 6 4}, 2-12$

10. Mare, L., and Trinchera, M. (2007) Comparative analysis of retroviral and native promoters driving expression of betal,3galactosyltransferase beta3Gal-T5 in human and mouse tissues. J. Biol. Chem. 282, 49-57

11. Caretti, A., Sirchia, S. M., Tabano, S., Zulueta, A., Dall'Olio, F., and Trinchera, M. (2012) DNA methylation and histone modifications modulate the $\beta 1,3$ galactosyltransferase $\beta 3 \mathrm{Gal}-\mathrm{T} 5$ native promoter in cancer cells. Int. J. Biochem. Cell Biol. 44, 84-90

12. Wu, G. D., Chen, L., Forslund, K., and Traber, P. G. (1994) Hepatocyte nuclear factor-1 alpha (HNF-1 alpha) and HNF-1 beta regulate transcription via two elements in an intestinespecific promoter. J. Biol. Chem. 269, 17080-17085

13. Mare, L., and Trinchera, M. (2004) Suppression of $\beta 1$,3galactosyltransferase $\beta 3 \mathrm{Gal}-\mathrm{T} 5$ in cancer cells reduces sialyl-Lewis a and enhances poly $\mathrm{N}$-acetyllactosamines and sialyl-Lewis $\mathrm{x}$ on $\mathrm{O}$ glycans. Eur. J. Biochem. 271, 186-194

14. Keller, M. S., Ezaki, T., Guo, R. J., and Lynch, J. P. (2004) Cdx1 or Cdx2 expression activates E-cadherin-mediated cell-cell ad- 
hesion and compaction in human COLO 205 cells. Am. J. Physiol. Gastrointest. Liver Physiol. 287, G104-G114

15. Trinchera, M., Malagolini, N., Chiricolo, M., Santini, D., Minni, F., Caretti, A., and Dall'Olio, F. (2011) The biosynthesis of the selectin-ligand sialyl Lewis $\mathrm{x}$ in colorectal cancer tissues is regulated by fucosyltransferase VI and can be inhibited by an RNA interference-based approach. Int. J. Biochem. Cell Biol. 43, $130-139$

16. Pilozzi, E., Onelli, M. R., Ziparo, V., Mercantini, P., and Ruco, L. (2004) CDX1 expression is reduced in colorectal carcinoma and is associated with promoter hypermethylation. J. Pathol. 204, 289-295

17. Wicking, C., Simms, L. A., Evans, T., Walsh, M., Chawengsaksophak, K., Beck, F., Chenevix-Trench, G., Young, J., Jass, J., Leggett, B., and Wainwright, B. (1998) CDX2, a human homologue of Drosophila caudal, is mutated in both alleles in a replication error positive colorectal cancer. Oncogene 17, 657659

18. Caretti, A., Bianciardi, P., Sala, G., Terruzzi, C., Lucchina, F., and Samaja, M. (2010) Supplementation of creatine and ribose prevents apoptosis in ischemic cardiomyocytes. Cell. Physiol. Biochem. 26, 831-838

19. Gilliland, G., Perrin, S., Blanchard, K., and Bunn, H. F. (1990) Analysis of cytokine mRNA and DNA: detection and quantitation by competitive polymerase chain reaction. Proc. Natl. Acad. Sci. U. S. A. 87, 2725-2729

20. Suh, E., Chen, L., Taylor, J., and Traber, P. G. (1994) A homeodomain protein related to caudal regulates intestinespecific gene transcription. Mol. Cell. Biol. 14, 7340-7351

21. D'Angelo, A., Bluteau, O., Garcia-Gonzalez, M. A., Gresh, L., Doyen, A., Garbay, S., Robine, S., and Pontoglio, M. (2010) Hepatocyte nuclear factor lalpha and beta control terminal differentiation and cell fate commitment in the gut epithelium. Development 137, 1573-1582

22. Pontoglio, M. (2000) Hepatocyte nuclear factor 1, a transcription factor at the crossroads of glucose homeostasis. J. Am. Soc. Nephrol. 11, S140-S143
23. Gama-Sosa, M. A., Slagel, V. A., Trewyn, R. W., Oxenhandler, R., Kuo, K. C., Gehrke, C. W., and Ehrlich, M. (1983) The 5-methylcytosine content of DNA from human tumors. Nucleic Acids Res. 11, 6883-6894

24. Ehrlich, M. (2002) DNA methylation in cancer: too much, but also too little. Oncogene 21, 5400-5413

25. Ehrlich, M. (2009) DNA hypomethylation in cancer cells. Epigenomics. 1, 239-259

26. Aran, D., Sabato, S., and Hellman, A. (2013) DNA methylation of distal regulatory sites characterizes dysregulation of cancer genes. Genome Biol. 14, R21

27. Irizarry, R. A., Ladd-Acosta, C., Wen, B., Wu, Z., Montano, C., Onyango, P., Cui, H., Gabo, K., Rongione, M., Webster, M., Ji, H., Potash, J. B., Sabunciyan, S., and Feinberg, A. P. (2009) The human colon cancer methylome shows similar hypo- and hypermethylation at conserved tissue-specific CpG island shores. Nat. Genet. 41, 178-186

28. Kulis, M., Queirós, A. C., Beekman, R., and Martín-Subero, J. I. (2013) Intragenic DNA methylation in transcriptional regulation, normal differentiation and cancer [E-pub ahead of print] Biochim. Biophys. Acta 10.1016/j.bbagrm.2013.08.001

29. Aran, D., and Hellman, A. (2013) DNA methylation of transcriptional enhancers and cancer predisposition. Cell 154, 11-13

30. Kikuchi, R., Yagi, S., Kusuhara, H., Imai, S., Sugiyama, Y., and Shiota, K. (2010) Genome-wide analysis of epigenetic signatures for kidney-specific transporters. Kidney Int. 78, 569-577

31. Kheradpour, P., Ernst, J., Melnikov, A., Rogov, P., Wang, L., Zhang, X., Alston, J., Mikkelsen, T. S., and Kellis, M. (2013) Systematic dissection of regulatory motifs in 2000 predicted human enhancers using a massively parallel reporter assay. Genome Res. 23, 800-811

32. Rebollo, R., Romanish, M. T., and Mager, D. L. (2012) Transposable elements: an abundant and natural source of regulatory sequences for host genes. Annu. Rev. Genet. 46, 21-42

Received for publication August 12, 2013. Accepted for publication October 3, 2013. 